\title{
SPARSE GRAPH SIGNAL RECONSTRUCTION AND IMAGE PROCESSING ON CIRCULANT GRAPHS
}

\author{
Madeleine S. Kotzagiannidis, and Pier Luigi Dragotti \\ Department of Electrical and Electronic Engineering \\ Imperial College London \\ London, UK \\ \{m.kotzagiannidis13, p.dragotti\}@imperial.ac.uk
}

\begin{abstract}
In this work, we present extensions of the framework of sampling and reconstructing signals with a finite rate of innovation (FRI) to the graph domain, by tackling the problem of $K$-sparse graph signal reconstruction on perturbed circulant graphs, simulating network clusters within a large network. Given a dimensionality-reduced approximation of the GFT of the original graph signal, we develop a reconstruction approach, whereby, we operate on each subgraph individually using a set of approximation and denoising schemes. In particular, we employ a variation of Prony's method with Cadzow's algorithm, and further iterative denoising, which can lead to perfect reconstruction. In addition, we extend the application of recently developed circulant graph-wavelet filterbanks to images featuring patterns, in a novel model inspired by image segmentation, which involves a localized operation of the graph wavelet transform on individual segments of homogeneous intensity content, employing the nearest circulant matrix approximation scheme. The proposed method outperforms traditional methods in the classical domain in nonlinear approximation performance. We give preliminary results and discuss generalizations to arbitrary graphs.
\end{abstract}

Index Terms - finite rate of innovation, graph wavelet filterbank, circulant graph, image approximation

\section{INTRODUCTION}

Graphs, as high-dimensional (and often sparse) dependency structures, have become an increasingly favorable tool for the representation and processing of large data sets, primarily due to their potential to capture complexity beyond the classical domain. This has inspired the development of the field of Graph Signal Processing, as a counterpiece to traditional signal processing, with the aim of establishing comparable properties, operations and concepts in the graph domain. These range from basic operations such as modulation of a graph signal $([1],[2])$, a variety of graph-dependent downsampling strategies ([1],[2], [3]) up to more sophisticated constructions of graph wavelets ([4], [5]) and associated filterbanks ([6], [7], [8]), satisfying a set of properties equivalent to the classical domain, with applications ([9], [10]), and further theoretical investigations [11]. A major appeal of this emerging field is the ability to capture and incorporate additional information about the graph signal into the graph (such as similarity between sample values), yet also represents one of its challenges of finding adequate equivalencies to the classical domain without neglecting the newly arising data dependencies [1].

This work seeks to explore the concept of sparsity (and compressibility) on graphs, which includes sparsity in the vertex domain as well as in the graph wavelet domain. Hereby, we devote our attention to the class of circulant graphs, due to their particularly convenient properties, which facilitate e.g. downsampling and shifting operations ([2], [8], [12]). We first present extensions of the framework of reconstructing signals with a finite rate of innovation [13] to the graph domain in a novel model for the $K$-sparse graph signal recovery on network clusters. In the context of high-dimensional networks, we propose to model clusters as (un-)weighted and undirected circulant subgraphs, which are linked via inter-connecting edges on a main graph $G=(V, E)$, and are subject to perturbations in form of the addition and/or removal of randomly chosen edges. The graph spectral representation, or Graph Fourier Transform (GFT), on undirected, circulant graphs, is, up to a permutation, given by the DFT-matrix. In light of this, we present a variation of Prony's method [13] in a novel model of blockwise reconstruction operations with dimensionality reduction. We give preliminary results and further discuss the generalization to arbitrary graphs based on the nearest circulant approximation scheme ([14], [15]).

In addition, we extend the recently developed circulant spline-like graph wavelet filterbanks ([2], [8], [12]) to applications in image processing, particularly for images featuring sharp edges in patterns, in a novel framework based on image segmentation. Hereby, we initially apply a graph cut [16] on a given image to separate homogeneous regions from a pattern, and subsequently compute the nearest circulant graph approximations to the resulting subgraphs, using the aforementioned scheme, in order to construct circulant graphwavelet filterbanks localized to the image segments. We give preliminary results based on both artificial and realistic image patches, and discuss further extensions.

This paper is organized as follows: beginning with a brief summary of the background theory in Section 2, we present our novel framework for the reconstruction of sparse graph signals on (perturbed) circulant graphs in Section 3, followed by Section 4, which discusses the application of the aforementioned circulant graph wavelet filterbanks to images within our novel framework inspired by image segmentation. Section 5 contains preliminary experimental results for the methods developed in the previous sections, and in Section 6 we make concluding remarks.

\section{BACKGROUND}

In the following, we provide a brief overview of basic results in classical and graph signal processing as well as general matrix theory, which we will draw on in the main body of this work. 


\subsection{Graph Signal Processing and Circulant Graphs}

In the course of this paper, we consider the example of an undirected, (un-)weighted connected graph $G=(V, E)$, without selfloops, defined by a vertex set $V,|V|=N$, and an edge set $E$. The connectedness of $G$ is given by its adjacency matrix $\mathbf{A}$, with entries $A_{i, j}>0$ if there is an edge between nodes $i$ and $j$, and $A_{i, j}=0$ otherwise, and its degree matrix $\mathbf{D}$, which is diagonal with entries $D_{i, i}=\sum_{j} A_{i, j}$. The non-normalized graph Laplacian matrix $\mathbf{L}=\mathbf{D}-\mathbf{A}$ of $G$, which we will focus on in this work, has a complete set of orthonormal eigenvectors $\left\{\mathbf{u}_{l}\right\}_{l=0}^{N-1}$, with corresponding ordered, nonnegative eigenvalues $\left\{\lambda_{l}\right\}_{l=0}^{N-1}$.

A graph signal $\mathbf{x}$ is a real-valued scalar function defined on the vertices of a graph $G$, with sample value $x(i)$ at node $i[1]$. Analogously to the classical domain, one can define the Graph Fourier Transform (GFT) $\mathbf{X}^{G}$ of a graph signal $\mathbf{x}$, as the projection onto the graph Laplacian eigenbasis $\mathbf{U}=\left[\mathbf{u}_{0}|\cdots| \mathbf{u}_{N-1}\right]: \mathbf{X}^{G}=\mathbf{U}^{H} \mathbf{x}$, where $H$ denotes the Hermitian transpose [1].

The class of circulant graphs has been noted for its set of properties, which facilitate a number of analogous traditional signal processing notions, including linear shifting, convolution, and most importantly, downsampling strategies.

A graph $G$ is circulant with respect to a generating set $S=$ $\left\{s_{1}, \ldots, s_{M}\right\}$, with $0<s_{k} \leq N-1$ if there exists an edge between nodes $\left(i,\left(i+s_{k}\right)_{N}\right)$, for every $s_{k} \in S$ [2]. Downsampling on a circulant graph $G$ can be performed with respect to any element in its generating set $S$; for instance, to downsample a graph signal $\mathbf{x}$ by two with respect to $s=1$ is equivalent to keeping every other node [2]. In [8], a set of filters defined on circulant graphs were introduced, making up the so-called spline-like graph wavelet filterbank, which incorporates critical-sampling, and perfect reconstruction properties. The low-and highpass filters, which take (weighted) averages of neighboring nodes at 1-hop distances, are defined as:

$$
\begin{aligned}
\mathbf{H}_{L P} & =\frac{1}{2}\left(\mathbf{I}_{N}+\mathbf{A} \frac{1}{d}\right) \\
\mathbf{H}_{H P} & =\frac{1}{2}\left(\mathbf{I}_{N}-\mathbf{A} \frac{1}{d}\right)
\end{aligned}
$$

where $d$ represents the degree per node. For the filterbank to be invertible it is required that at least one node retains the LP component, while the complementary set of nodes are in the HP branch; this result applies to both weighted and unweighted undirected graphs and the proof can be found in [12]. Multiscale analysis can be conducted by iterating the designed filters on the respective downsampled LP branches, whereby proposed reconnection strategies for downsampled graphs include the Kron-reduction [12].

\subsection{FRI signals and Prony's method}

In classical sampling theory, it has been established that a certain class of non-bandlimited signals with a finite rate of innovation, known as FRI-signals, can be sampled and perfectly reconstructed using kernels of compact support, which satisfy certain Strang-Fix conditions, and a local reconstruction algorithm (Prony's method) [13]. In the discrete time domain, let a signal $\mathbf{x} \in \mathbb{R}^{N}$ be K-sparse, and define the measurement vector $\mathbf{y}$ in the Fourier domain, such that $\mathbf{y}=\mathbf{F x}$, where $\mathbf{F} \in \mathbb{C}^{N \times N}$ is the DFT-matrix, and $\mathbf{x} \in \mathbb{R}^{N}$, $\|\mathbf{x}\|_{0}=K$. Then the signal samples $y_{n}$ are given by

$$
y_{n}=\frac{1}{\sqrt{N}} \sum_{k=0}^{K-1} x_{c_{k}} e^{-i 2 \pi c_{k} n / N}=\sum_{k=0}^{K-1} \alpha_{k} u_{k}^{n}
$$

where $x_{c_{k}}$ is the weight of $\mathbf{x}$ at index $c_{k}$, and $\alpha_{k}=x_{c_{k}} / \sqrt{N}$ and $u_{k}=e^{-i 2 \pi c_{k} n / N}$ represent the amplitudes and locations respectively. The vector $\mathbf{x}$ can then be perfectly reconstructed based on $M=2 K$ consecutive sample values of $\mathbf{y}$ using Prony's method. If there is noise present in the signal, such as in form of additive Gaussian noise $\mathbf{n}$, giving $\hat{\mathbf{y}}=\mathbf{y}+\mathbf{n}$, we require a larger number of samples $M \geq 2 K$ as well as need to apply denoising schemes to achieve perfect reconstruction; hereby, Cadzow's algorithm has been favorably employed [17].

\subsection{Circulant Matrix Theory}

In a realistic setting, the graph at hand might not be circulant, which requires a means to detect the nearest circulant structure as determined by a given error norm. Therefore, we resort to the so-called Chan circulant matrix ([14], [15]), which gives the nearest circulant matrix $\mathbf{C} \in \mathbb{R}^{N \times N}$ to a given adjacency matrix $\mathbf{A} \in \mathbb{R}^{N \times N}$ in Frobenius norm $\|\mathbf{A}-\mathbf{C}\|_{F}$, by averaging over its diagonals. Let $\Pi$ be a circulant matrix with first row $\pi=\left[\begin{array}{lllll}0 & 1 & 0 & \ldots & 0\end{array}\right]$. Then, the nearest circulant matrix $\mathbf{C}$, with first row $\mathbf{c}=\left[\begin{array}{llll}c_{0} & c_{1} \ldots c_{N-1}\end{array}\right]$, to a given $\mathbf{A}$ is determined by the following Frobenius inner product:

$$
c_{k}:=\frac{1}{N}\left\langle\mathbf{A}, \boldsymbol{\Pi}^{k}\right\rangle_{F}=\operatorname{tr}\left(\mathbf{A}^{T} \boldsymbol{\Pi}^{k}\right), \quad k=0, \ldots, N-1
$$

For undirected, weighted graphs, we require the weights to satisfy symmetry and circularity, i.e. when $N$ is even, the first row of the adjacency matrix $\mathbf{W}$ needs to be of the form

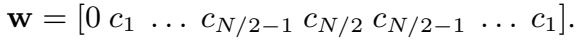

The GFT on circulant graphs, is, up to a permutation, given by the DFT-matrix due to the circulant structure of the graph Laplacian [12]. However, with eigenvalue multiplicities occurring, the eigenbasis is not unique. To the best of our knowledge, there does not exist a systematic way to detect the eigenvalue multiplicity distribution of a circulant graph $G$ with an arbitrary generating set $S$, except by the exhaustive search approach; one can merely infer basic results on the occurrence of odd and even multiplicities of eigenvalues of circulant matrices, as stated in [18].

\section{SPARSE SIGNAL RECONSTRUCTION ON CIRCULANT GRAPHS}

In the context of high-dimensional networks, we wish to consider network-clusters, representing groups of strongly connected entities within a large network (e.g. friends in a social network). Hereby, we propose to model the clusters as undirected and (un-)weighted circulant subgraphs, which are linked via few inter-connecting edges on a main graph $G=(V, E)$, and are subject to perturbations in form of the addition and/or removal of randomly chosen edges, to simulate the randomness and complexity occuring in real-world network clusters. In the following, we present a novel model for the $K$-sparse graph signal reconstruction on perturbed circulant subgraphs of such networks as an extension of the framework of sampling and reconstructing FRI-signals in the classical domain.

As the underlying graph structure, and therefore its associated GFT, is fixed, one can sample a set of distinct signals $\left\{\mathbf{x}_{i}\right\}_{i=1}^{m}$, of the same dimension $N$ and sparsity $K$, on $G$, which gives rise to a novel framework of sparse signal sampling and reconstruction on graphs: Given a partial GFT-matrix $\mathbf{U}$ of the underlying graph and the dimensionality-reduced measurement vector(s) of the form $\mathbf{y}=$ $\mathbf{C U}^{H} \mathbf{x}$, where $\mathbf{C}$ represents an appropriate coefficient matrix, we can reconstruct the (set of) sparse signal(s), which are represented on 
$G$. While it is possible to operate on the entire graph at once, using a blockwise matrix operation scheme, which we omit here for brevity, we choose to operate on each subgraph individually by performing normalized graph cuts [16] on inter-connecting edges between perturbed circulant subgraphs, and subsequently apply our scheme on each.

We begin the extension to the graph domain by introducing a coefficient matrix $\mathbf{C}$, in order to impose a permutation, and, if the graph at hand is not circulant, approximate the DFT-matrix by the given GFT-basis of the graph $G$. In particular, given a subset of $P$ eigenvectors $\mathbf{U}_{P}$ of the GFT-basis $\mathbf{U}=\left[\mathbf{u}_{0}|\cdots| \mathbf{u}_{N-1}\right]$, as defined for a clustered subgraph $G$, and the dimensionality-reduced measurement vector $\mathbf{y}=\mathbf{C U}_{P}^{H} \mathbf{x}, \mathbf{y} \in \mathbb{C}^{M}$, where $\mathbf{C} \in \mathbb{C}^{M \times P}$ and $M<P<N$, we can perfectly reconstruct a $K$-sparse graph signal $\mathbf{x} \in \mathbb{R}^{N},\|\mathbf{x}\|_{0}=K$, for suitably chosen parameters $M$ and $P$. Hereby, the coefficient matrix $\mathbf{C}$ is constructed to approximate the first $M$ rows of the DFT-matrix $\mathbf{F}_{M} \approx \mathbf{C U}_{P}^{H}$. We require $\mathbf{C}$ to be fat with the dimensionality offset $P=2 M-1$ in the case of least possible graph Laplacian eigenvalue multiplicities; thus, in an ideal scenario of a circulant graph $G$ with the maximum multiplicity of $m_{i}=2$ per eigenvalue (except for $\lambda_{0}=0$, and $\lambda_{N-1}$ if $N$ is even, where the location of the latter in the spectrum may vary), we can perfectly reconstruct a sparse signal x defined on the vertices of $G$, requiring only $P=2 * 2 K-1=4 K-1$ sample values. This is satisfied by the simple cycle, for instance.

In our proposed scheme, we initially apply Cadzow's denoising algorithm [17] on the given vector $\mathbf{y}$, followed by Prony's method, so as to recover a first estimate $\hat{\mathbf{x}}$ of the desired $K$-sparse vector. Subsequently, we proceed with an iterative denoising scheme, which utilizes the error matrix $\mathbf{E}=\mathbf{C} \mathbf{U}_{P}^{H}-\mathbf{F}_{M}$ to effectively remove the perturbation noise based on the current estimate of $\mathbf{x}$ from the measurement vector at iteration $i: \mathbf{y}^{i+1}=\mathbf{y}-\mathbf{E} \hat{\mathbf{x}}^{i}$. Hereby, we

\footnotetext{
Algorithm 1 Sparse Graph Signal Reconstruction

1: Input: Adjacency matrix $\mathbf{A}$ of graph $G$, and graph signal $\mathbf{x}$, with $\|\mathbf{x}\|_{0}=K$

2: Decompose $G$ into $T$ disconnected subgraphs $\left\{G_{l}\right\}_{l=1}^{T}$. Project
} the graph signal values onto the associated nodes, resulting in $T$ signals $\left\{\mathbf{x}_{l}\right\}_{l=1}^{T}$ with new sparsity $\left\{K_{l}\right\}_{l=1}^{T}$. Apply the following scheme(s) on each subgraph individually

3: Option 1: Compute the nearest circulant $\tilde{\mathbf{A}}_{l}$ to $\mathbf{A}_{l}$, via (4). Sample $\mathbf{x}_{l}$ on the vertices of $\tilde{G}_{l}$, and, if required, impose a permutation $\sigma_{\tilde{\Lambda}_{j}}$ on the GFT basis $\tilde{\mathbf{U}}$ of $\tilde{G}_{l}$, according to the eigenvalue sequence $\tilde{\Lambda}_{j}$ obtained by taking the DFT of the first row of the graph Laplacian of $\tilde{G}_{l}$, and arranging eigenvalues of the same subspace $j$ together. Compute $\mathbf{C}$ via LS: $\mathbf{C}^{T}=\left(\tilde{\mathbf{U}}_{P}^{H}\right)^{T} \backslash \mathbf{F}_{M}^{T}$. We only require $P=4 K_{l}-1$ consecutive samples for perfect reconstruction, at best. Ensure that $\tilde{G}_{l}$ has the required minimum of multiplicities (or adjust $P$ accordingly). Store vector $\hat{\mathbf{y}}=\tilde{\mathbf{U}}_{P}^{H} \mathbf{x}_{l} \in \mathbb{C}^{P}$

4: Option 2: Model the graph as circulant with a perturbation, by first computing the nearest circulant $\tilde{\mathbf{A}}_{l}$ to the given $\mathbf{A}_{l}$, and imposing the permutation $\sigma_{\tilde{\Lambda}_{j}}$ on $\mathbf{U}$ of $G_{l}$, if required. Compute $\mathbf{C}$, and create $\mathbf{y}=\mathbf{C} \mathbf{U}_{P}^{H} \mathbf{x}_{l}$ for appropriate $M$, and $P=f(M)$ (depending on the multiplicities of $\tilde{G}_{l}$ ). Apply the proposed denoising scheme at $P \geq 4 K_{l}-1 \geq f(M)$ :

5: Apply Cadzow's algorithm on $\mathbf{y}$, followed by Prony's method to recover $\hat{\mathbf{x}}_{l}$

6: Do further iterative denoising, $\mathbf{y}^{i+1}=\mathbf{y}-\mathbf{E} \hat{\mathbf{x}}_{l}^{i}$, as required, and repeat 5 . Store vector $\hat{\mathbf{y}}=\mathbf{U}_{P}^{H} \mathbf{x}_{l} \in \mathbb{C}^{P}$. reduce the error caused by initial estimates $\hat{\mathbf{x}}$ in a setting of highly irregular and/or localized noise. It has been established that for a sufficiently large number of given samples $M$, and an iteration number $i \geq 5$, we can achieve perfect reconstruction. Overall, we propose the approaches summarized in Algorithm 1 for sparse signal reconstruction with dimensionality reduction. We further note that Option 2 applies only to a subset of graphs with certain circulant generating sets, including e.g. the simple cycle with $S=\{1\}$. For the complementary set, we observe a pattern of highly localized noise under perturbation, caused by the permutation/approximation steps, which can lead to destructive performance in the reconstruction process. In addition, when the perturbation is more invasive relative to the graph at hand, further permutation schemes may need to be applied due to subspace swap phenomena, which we omit here for brevity.

\section{CIRCULANT GRAPH WAVELETS FOR NON-LINEAR IMAGE APPROXIMATION}

Having previously explored sparse graph signals, we now proceed to investigate the concept of sparsity in the graph wavelet domain, as arising from smooth graph signals with discontinuities relative to the graph at hand. There exist graph-specific interpretations of the classical concept of compressibility of signals, as discussed in [19], including a measure for the smoothness of a graph signal.

We wish to consider the realm of 2D images that incorporate irregularly shaped, sharp discontinuities between homogeneous regions, or more precisely, featuring distinct alternating patterns, such as stripes. By exploiting the flexibility of graph-based constructions, which facilitate operations across irregularly shaped image segments, as opposed to the rather 'stiff' operations across rows and columns of 2D transforms in the classical domain, we formulate a novel framework of localized graph wavelet transformations inspired by image segmentation, which allows the construction of graph wavelet filterbanks on individual image segments. Hereby, we refer to the circulant spline-like graph wavelet filterbank introduced in [8]. As outlined in Algorithm 2, we initially perform a graph cut [16] on the given image, in order to obtain homogeneous image regions,

\begin{tabular}{l}
\hline Algorithm 2 Image Processing on Circulant Graphs \\
\hline 1: Input: Grayscale Image A \\
2: Construct an undirected, weighted graph $G$ based on A, where
\end{tabular}

each node represents a pixel, using a similarity measure of the form $w_{i, j}=e^{-\frac{\left|I_{i}-I_{j}\right|^{2}}{\sigma_{I}^{2}}} e^{-\frac{|| p_{i}-p_{j} \|\left.\right|_{2} ^{2}}{\sigma_{P}^{2}}}$ between nodes $i$ and $j$, where $p$ denotes the Euclidean distance function, and $I$ gives the intensity value. Let the vectorized form $\mathbf{x}$ of the intensity values of $\mathbf{A}$ denote the graph signal on $G$, with $x(i)=I(i)$ at node $i$

3: Perform a normalized graph cut on $G$, with weighted adjacency matrix $\mathbf{W}$, resulting in two subgraphs with respective adjacency matrices $\mathbf{W}_{1}$ and $\mathbf{W}_{2}$, and sub-graph signals $\mathbf{x}_{1}$ and $\mathbf{x}_{2}$

4: Compute the nearest circulant approximations $\tilde{\mathbf{W}}_{1}, \tilde{\mathbf{W}}_{2}$ to the weighted adjacency matrices of the subgraphs. If $\mathbf{W}_{i}$ is sparse, apply the RCM-algorithm [20] to obtain a matrix with smaller bandwidth under a different node ordering, beforehand.

5: Construct the circulant graph wavelet transform on both subgraphs, and apply it on the respective sub-graph signals. Iterate accordingly on the LP-branches, for a multiscale representation. 6: Iterate further graph cuts as appropriate

which are not necessarily connected in the original image. We proceed to construct and apply circulant graph wavelets on the individ- 
ual regions, with the aim to obtain a sparse representation, since the graph-Laplacian-based construction of the filters leads to complete annihilation of constant graph signals in the HP-branch. Hereby, we downsample with respect to the element $s=1$, and choose the nearest circulant approximation as a reconnection scheme. For instance, in case of a binary simple stripe pattern, we can achieve perfect reconstruction in non-linear approximation at a small number of non-zero coefficients due to complete annihilation in the HP-branch. By initially not imposing any sparsity constraints on the graphs involved, the resulting circulant subgraphs are weighted and complete. In order to obtain a more localized construction of the graph wavelet with respect to the image segment, as opposed to the entire image, one can explore further sparsification schemes of the resulting subgraphs, which we omit for brevity.

\section{EXPERIMENTAL RESULTS}

\subsection{Sparse Graph Signal Reconstruction}

We consider the example of a simple cycle subject to perturbations in form of two additional, randomly distributed edges, and apply Option 2 in Algorithm 1. Figure 1 illustrates the reconstruction performance per iteration in form of the average location error between the estimated and true entry locations of the randomly generated sparse vector $\mathbf{x}$ averaged over 100 trials, given the dimensionality reduced measurement vector $\mathbf{y} \in \mathbb{C}^{M}$, and $P=2 M-1$ samples.

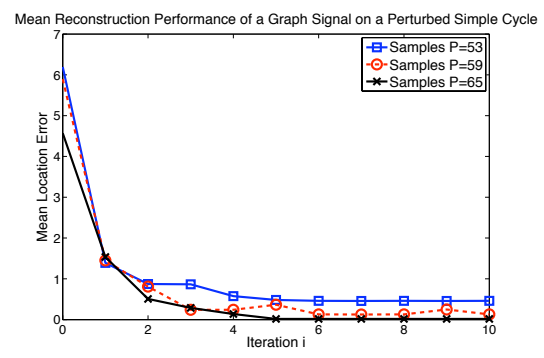

Fig. 1. Reconstruction Performance on Perturbed Simple Cycle $(N=256)$, for 100 randomly generated sparse signals $\mathbf{x}_{l}(K=4$, minimum entry separation of 3 )

\subsection{Image Approximation}

We demonstrate the performance of our developed method of the graph-cut based circulant graph-wavelet transform (GWT) by comparing it to the classical 2D Haar transform and the 2D biorthogonal spline transform (with 2 vanishing moments), each with 5 levels of decompositions. The initial graph $G$ results in weighted, complete subgraphs. Figures 2 and 3 compare the performance measured as the relative Frobenius-norm error for an artificial and a real image patch taken from 'cameraman', respectively. It becomes evident that our proposed method can outperform traditional methods at a small number of non-zero coefficients, especially when employing a greater number of graph cuts.

\section{CONCLUSIONS AND FUTURE WORK}

Preliminary results arising from the novel framework of FRIsampling and reconstruction on graphs reveal interesting analogies

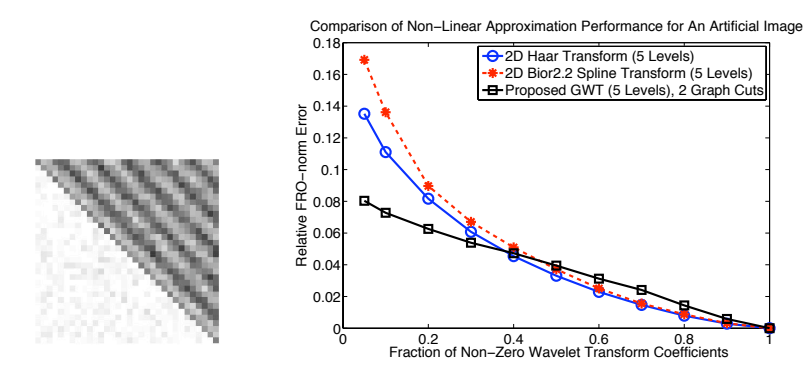

Fig. 2. Non-linear Approximation Performance for a $32 \times 32$ Artificial Image Patch
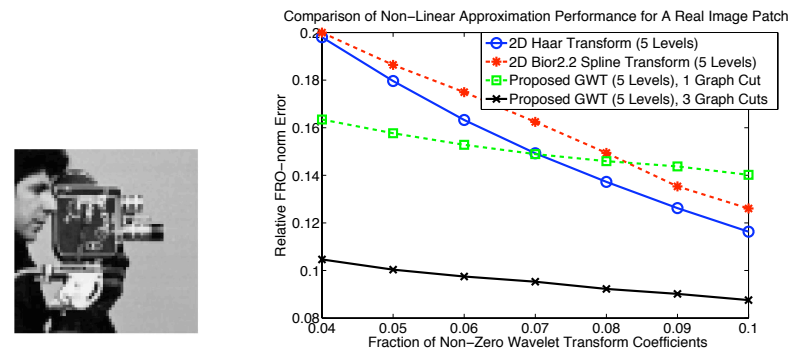

Fig. 3. Non-linear Approximation Performance for a $64 \times 64$ Real Image Patch

to the classical domain with promising potential of dimensionality reduction. A deeper understanding of the specific circulant generating sets satisfying these properties, and the overall graph-theoretic interpretation of our approximation scheme need to be explored further. The combination of circulant graph wavelet filterbanks with graph cuts proves as a promising new venture with clear structural advantages over classical methods for image approximation. We aim to continue to explore variations, which are more localized in the graph domain as well as annihilate higher-order graph signals.

\section{REFERENCES}

[1] D. I. Shuman, S. K. Narang, P. Frossard, A. Ortega, and P. Vandergheynst, "The emerging field of signal processing on graphs: Extending high-dimensional data analysis to networks and other irregular domains", SP Magazine, IEEE, vol. 30, no. 3, pp. 8398, 2013.

[2] Y. N. Ekambaram, G. Fanti, B. Ayazifar, and K. Ramchandran,"Circulant structures and graph signal processing", in Proc. of the IEEE International Conference on Image Processing (IC/P), pp. 834-838, 2013.

[3] S. Narang and A. Ortega, "Downsampling graphs using spectral theory", in Proc. Int. Conf. Acoust., Speech, Signal Process. (ICASSP), pp. 4208-4211, 2011.

[4] D. Hammond, P. Vandergheynst, and R. Gribonval, "Wavelets on Graphs via Spectral Graph Theory", Appl. Comput. Harm. Anal., vol. 30, no. 2, pp. 129-150, March 2011.

[5] R. Coifman and M. Maggioni, "Diffusion Wavelets", Applied and Computational Harmonic Analysis, vol. 21, no. 1, pp. 5394, July 2006. 
[6] S. Narang, and A. Ortega, "Perfect Reconstruction Two-Channel Wavelet Filter Banks for Graph Structured Data", IEEE Transactions on Signal Processing, vol. 60, issue 6, pp. 2786-2799, February 2012

[7] S. K. Narang and A. Ortega, "Multi-dimensional separable critically sampled wavelet filterbanks on arbitrary graphs", Proc. ICASSP, pp. 254 -262, March 2012

[8] V. N. Ekambaram, G. Fanti, B. Ayazifar, and K. Ramchandran, "Critically-Sampled Perfect-Reconstruction Spline-Wavelet Filterbanks for Graph Signals", IEEE GlobalSIP, pp. 475-478, 2013.

[9] S. K. Narang, Y. H. Chao and A. Ortega, "Graph-wavelet filterbanks for edge-aware image processing", Proc. IEEE SSPWorkshop, pp.141-144, August 2012.

[10] Y. Wang, A. Ortega, D. Tian, and A. Vetro, "A graphbased joint bilateral approach for depth enhancement", International Conference on Acoustics, Speech and Signal Processing, (ICASSP), pp. 885-889, 2014

[11] M. Rabbat and V. Gripon, "Towards a Spectral Characterization of Signals Supported on Small-World Networks", in Proc. Int. Conf. Acoust., Speech, Signal Process. (ICASSP), pp. 47934797, May 2014.

[12] V. Ekambaram, "Graph Structured Data Viewed Through a Fourier Lens", PhD Thesis, EECS Department, University of California, Berkeley, December 2013.

[13] T. Blu, P.L. Dragotti, M. Vetterli, P. Marziliano and L. Coulot, "Sparse Sampling of Signal Innovations: Theory, Algorithms and Performance Bounds", IEEE Signal Processing Magazine, vol. 25, no. 2, pp. 31-40, March 2008.

[14] T. F. Chan, "An optimal circulant preconditioner for Toeplitz systems”, SIAMJ. Sci. Stat. Comp., vol. 9, no. 4, pp. 766-771, 1988.

[15] M. T. Chu, and R. J. Plemmons, "Real-Valued, Low Rank, Circulant Approximation", SIAM J. Matrix Anal. Appl., vol. 24, no. 3, pp. 645-659, March 2002.

[16] J. Shi and J. Malik, "Normalized Cuts and Image Segmentation", IEEE Transactions on Pattern Analysis and Machine Intelligence, vol. 22, no. 8, pp. 888-905, August 2000

[17] J. Cadzow, "Signal enhancement: A composite property mapping algorithm", IEEE Trans. Acoust., Speech, Signal Processing, vol. 36, no. 1, pp. 49-62, January 1988.

[18] G. Tee, "Eigenvectors of block circulant and alternating circulant matrices", New Zealand Journal of Mathematics, vol. 36, no. 8, pp. 195-211, January 2007.

[19] X. Zhu and M. Rabbat, "Approximating signals supported on graphs", Proc. IEEE Int. Conf. Acoust., Speech, Signal Process., pp. $3921-3924,2012$.

[20] E. Cuthill and J. McKee, "Reducing the bandwidth of sparse symmetric matrices", Proceedings of the 1969 24th National Conference, pp. 157-172, ACM, 1969. 